\title{
Fully Integrated PHEMT Voltage Controlled Oscillator with Very High Tuning Bandwidth
}

\author{
A. Megej, K. Beilenhoff ${ }^{\dagger}$, and H.L. Hartnagel \\ Institut für Hochfrequenztechnik, Technische Universität Darmstadt, Merckstraße 25, \\ D-64283 Darmstadt, Germany. E-mail: megej@tu-darmstadt.de
}

\begin{abstract}
Design concepts of fully monolithically integrated VCOs are presented that include special solutions for increasing the bandwidth of operation. Realised buffered VCO MMICs deliver RF power between 11.2 and $15.5 \mathrm{dBm}$ over the frequency range from 4.6 to $6.1 \mathrm{GHz}$. The relative bandwidth $\Delta f / f_{m}$ is more than $27 \%$, which is one of the highest values ever achieved with MMIC-VCOs using diode tuning.
\end{abstract}

\section{INTRODUCTION}

$\mathrm{T}$ HE LAST time has seen an increasing use of monolithic microwave integrated circuits (MMICs) for a variety of applications. The MMIC realisation offers, in comparison to a hybrid system, lower cost when manufactured in high quantities. MMICs are also well known for their excellent reproducibility, high reliability, and low value of parasitics. The use of MMICs in sensor, automotive, and other applications has created the demand on fully integrated broadband voltage controlled oscillators (VCOs).

The most wide-band MMIC VCO realisations make use of off-chip resonators for frequency tuning [e.g. 1,2]. The fully integrated circuits presented before [e.g. 3,4] exhibit a relative narrow bandwidth of frequency variation. The maximal relative bandwidth achieved with such monolithic diode-tuned VCOs has been reported to be as high as $14 \%$ [4].

Since common MMIC fabrication processes are usually optimised for high power or high frequency operation, the capacitance change available with diodes is of minor importance. However, for wide-band VCOs the ratio $C_{\max } / C_{\min }$ is the figure or merit, which is finally decisive for the frequency range covered by the VCO. Moreover, if the HEMT structures are used, the diodes available in the process are more or less degenerated, since the capacitance effect is mainly caused by modulation of the 2DEG.

On the other hand, the (P)HEMT (pseudomorphic high electron-mobility transistor) process is a standard one for high frequency applications. Its use is essential, if the

Dr. Beilenhoff is now with the United Monolithic Semiconductors S.A.S., ORSAY/FRANCE oscillator has to be employed on the same chip with amplifiers, mixers, and other sub-circuits to build a system with certain functionality.

This paper describes design concepts and the realisation of a very compact, wide-band MMIC VCO with integrated diodes employed for frequency tuning. A special emphasis is hereby laid on single design solutions such as resonator circuit design and oscillation transistor configuration, which help to increase the tuneable bandwidth of VCOs. Particularly, the resonator circuit configuration helps to increase the oscillation bandwidth.

We used for our wide-band circuit design the $0.25 \mu \mathrm{m}$ PHEMT PH25-process of UMS. The circuit realisation described in this paper provides oscillations within a wide range of $4.6-6.1 \mathrm{GHz}$, which amounts to the relative bandwidth of almost $\Delta f / f_{m}=28 \%$. The output power of the buffered VCO varies within the values of 11.2 and $15.5 \mathrm{dBm}$. The chip area used for the implementation of the VCO and buffer amplifier is $2.5 \times 1.0 \mathrm{~mm}^{2}$, whereby the $\mathrm{VCO}$ alone requires area with dimensions of only $1.1 \times 1.0 \mathrm{~mm}^{2}$.

\section{CiRcuit DESIGN}

The schematic diagram of the voltage controlled oscillator is shown in Fig. 1. The VCO employs a $6 \times 40 \mu \mathrm{m}$ PHEMT in common-source configuration. The capacitors $C_{F B}$ realises the feedback in order to achieve negative differential resistance at the input of transistor. This transistor configuration has often been used for oscillator design [e.g. 1,5,7] due to its excellent RF performance.

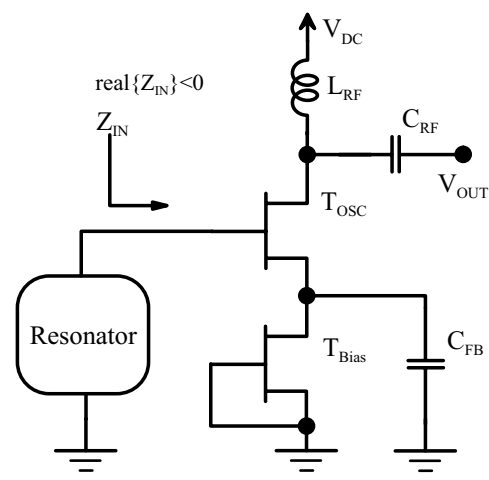

Fig. 1. Principle VCO schematic including biasing nework 


\section{A. Active Biasing Technique}

The disadvantage of the concept described above can be found in the very difficult bias supply. Usually, $\lambda / 4-$ transmission lines or spiral inductors are used to separate DC - and RF-path. These implementations require chiparea, which is often much larger than used by the active circuit itself. In particular, at relative low frequencies. Furthermore, the current limitation within integrated spiral inductors often requires the use of two components in parallel. This solution does not even help to reduce the chip area.

For the chosen oscillator-transistor configuration shown in Fig. 1, we need two DC networks: in the source and in the drain paths of the active element. Thus, the problems in connection with the DC-supply mentioned above double.

In our case, we used an active-biasing configuration in the source part of the oscillating transistor. For the biasing transistor, a very small $1 \times 30 \mu \mathrm{m}$ PHEMT was chosen. The gate voltage of the oscillating transistor $T_{O S C}$ is set to zero in order to reduce the design effort. The operation point of the transistor configuration changes, but its high-frequency behaviour is still suitable for oscillator design.

Using this kind of biasing, we reach different goals at the same time. On the one side, the biasing is now realised over a wide frequency range. The current consumed by the circuitry becomes very low due to the different bias point. This leads to the fact that the requirements to the current-handling capability of the spiral inductor employed in the drain path of the oscillating transistors are also low and that simplifies the design. Further, the DC- to RF efficiency of the design could be improved.

\section{B. Resonator Circuit Design}

As already mentioned above, the tuning bandwidth achievable with a specific transistor is mainly determined by the ranges of the reactance values exhibited by the resonator circuit. Hence, the design of this circuitry plays an important role for the final bandwidth of a VCO.

Usually, varactor diodes are used within resonator circuitry for frequency tuning. Nowadays, some MMIC VCO designs have been published, where active inductors are used for frequency tuning [e.g. 5,6]. This concept allows one to reach higher tuning bandwidth, but it has only been used for rather low frequencies.

As described above, real varactor diodes cannot be realised with the process used. Therefore, integrated planar diodes based on the PHEMT epitaxial structure are used as voltage-tuned reactance elements. The dimensions of these diodes are $10 \times 75 \mu \mathrm{m}$ ( 10 fingers with $75 \mu \mathrm{m}$ anode width each). The junction capacitance of these diodes changes with the applied DC-voltage within the range of $C \approx 500$ to $1600 \mathrm{fF}$. The low capacitance modulation ratio of $C_{\max } / C_{\min } \approx 3$ is a common value for such MMIC diodes.

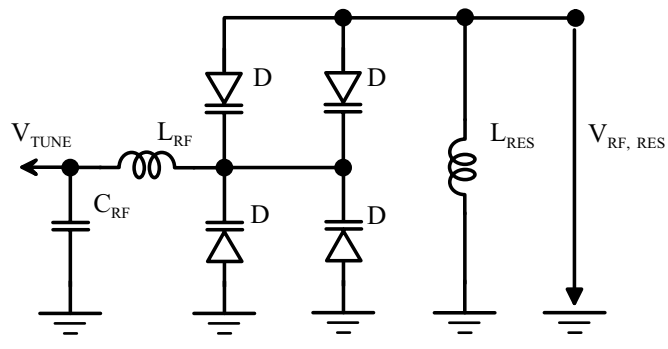

Fig. 2. Principle schematic of the resonator circuit

It is necessary to keep in mind that this value is calculated by small-signal analysis techniques. The higher the RF-voltage on the tuning elements, the lower the effective reactance modulation ratio. This effect is common for all the semiconductor devices acting as tuning elements. It causes a reduction of the diode capacitance modulation ratio and, hence, the maximal bandwidth of oscillations. To overcome this problem two diodes are connected in series. Two other serially connected diodes are introduced into the resonator circuit to obtain the desirable capacitance value. Figure 2 shows the resulting principle schematic of the resonator circuit employed. The inductance $L$ is used to fulfil the phase requirement of oscillation conditions at the input of the transistor. In this configuration, only half of the entire RF- voltage $\left(V_{R F}\right.$ in Fig. 1) is applied to each diode pair. Figure 3 demonstrates the value of diode capacitance calculated for one diode and for the series-parallel configuration using small- and large-signal analysis techniques $\left(V_{R F, R e s}=0.5 \mathrm{~V}\right)$. The simulations have also demonstrated that the bandwidth covered by this resonator is higher in comparison to the simple configuration. The measurements fully confirm this effect. A further advantage of this circuit is the fact that the bias supply to the tuning diodes can very easily be provided.

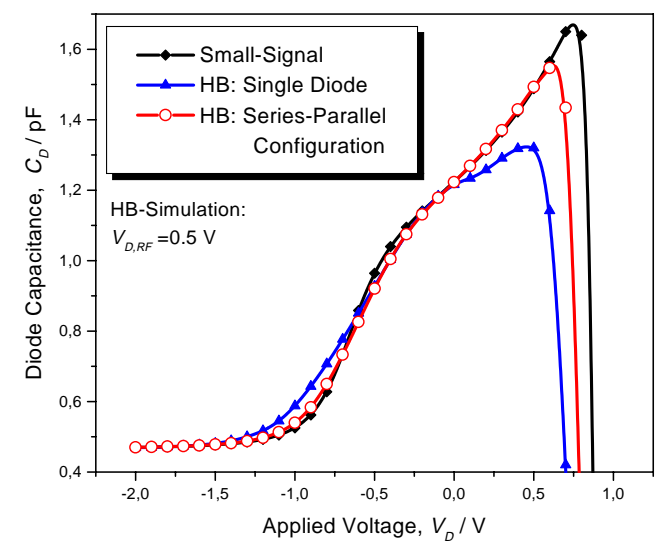

Fig. 3. Calculated diode capacitance for different configurations. Small-signal and HB (Harmonic Balance) Simulations 


\section{Buffer Amplifier Design}

The buffer amplifier was designed with the aim to reduce the influence of the load variation on the frequency of oscillations (load-pull effect). The input network of amplifiers was designed to provide a value of the impedance that helps to increase the overall bandwidth of oscillation. Table I shows the summary of simulated and measured amplifier characteristics.

TABLE I

SUMMARY OF CALCULATED AND MEASURED AMPLIFIER PERFORMANCE

\begin{tabular}{|l|c|c|c|}
\hline \multicolumn{2}{|c|}{} & Sim. & Meas. \\
\hline \hline Forw. gain & $G / \mathrm{dB}$ & $11.0-11.8$ & $10.6-11.8$ \\
\hline Rev. Gain & $a_{d, \max } / \mathrm{dB}$ & -24.3 & -25.0 \\
\hline Input. Match. & $\left|S_{11}\right|_{\max } / \mathrm{dB}$ & -17.0 & -15.6 \\
\hline
\end{tabular}

\section{RESULTS}

Using the technique described above, voltage controlled oscillators with and without buffer amplifiers were realised and characterised.

The on-wafer measurements were performed using spectrum analyser HP8565E and the network analyser system HP8510B. The following subsections describe the achieved results.

\section{A. Single $V C O$}

In order to investigate the influence of the proposed biasing solution on performance of a wide-band VCO the behaviour of two oscillator circuits were designed, simulated and laid out using HP-EESOF MDS. One design employs the active biasing described above, whereas the other circuitry was designed using common spiral inductors for DC-power supply. These circuits only distinguish in this point to ensure the comparability of the calculated results.

The calculated results of both the active- and passivebiased VCOs are listed in Table II. The measured performance of the practical realisation incorporating active biasing technique can also be found in this table.

The main advantages of the active biasing are as follows:

- significant reduction (about 35\%) of the chip-area used. The designed and processed circuit requires area of only $1.1 \times 1.0 \mathrm{~mm}^{2}$ in comparison to $1.3 \times 1.3 \mathrm{~mm}^{2}$ for the realisation with inductor-based DC-supply.

- the VCO with a PHEMT used for bias-supply proves to increase the bandwidth of this circuitry. This is mainly due to the fact that, in this case, the imaginary part of the negative differential resistance at the input varies less than in passive bias supply case. The bandwidth gain on the tuneable frequency range amounts to the value around $5 \%$.
TABLE II

CALCULATED AND MEASURED PERFORMANCE OF VCOS WITH DIFFERENT BIASING METHODS

\begin{tabular}{|l|c|c|c|}
\hline \multirow{2}{*}{ Biasing Method } & \multicolumn{2}{|c|}{ Active } & Passive \\
\cline { 2 - 4 } & Meas. & Sim. & Meas. \\
\hline \hline $\begin{array}{l}\text { Chip Area } \\
\text { A/mm }{ }^{2}\end{array}$ & $\mathbf{1 . 1} \times \mathbf{1 . 0}$ & $\mathbf{1 . 1} \times \mathbf{1 . 0}$ & $\mathbf{1 . 3} \times \mathbf{1 . 3}$ \\
\hline $\begin{array}{l}\text { Frequency } \\
f / G H z\end{array}$ & $4.69-6.0$ & $4.75-6.0$ & $4.83-5.83$ \\
\hline $\begin{array}{l}\text { Rel. Bandwidth } \\
\Delta f / f_{m} / \%\end{array}$ & $\mathbf{2 4 . 5}$ & $\mathbf{2 3 . 3}$ & $\mathbf{1 8 . 8}$ \\
\hline $\begin{array}{l}\text { Max. Efficiency } \\
\eta_{\text {max }} / \%\end{array}$ & $\mathbf{3 3 . 4}$ & $\mathbf{2 8 . 9}$ & $\mathbf{2 0 . 3}$ \\
\hline $\begin{array}{l}\text { Max. Power } \\
P_{\text {out }, \text { max }} / \mathrm{dBm}\end{array}$ & 7.0 & 6.6 & 12.7 \\
\hline $\begin{array}{l}\text { Drain Current } \\
I_{D} / \mathrm{mA}\end{array}$ & 7.6 & 8.0 & 46.0 \\
\hline
\end{tabular}

- further, the utilisation of the proposed biasing technique helps to increase the overall DC- to RF-efficiency of voltage controlled oscillators, although the absolute output power of the oscillator with spiral inductors within DC-networks is expected to be higher. Nevertheless, the drastic reduction of the drain current causes this effect. The DC-voltage applied to the both circuits investigated is set to a low value of $2 \mathrm{~V}$.

\section{B. Buffered Design}

This design is similar to the one described above. The only difference is the utilisation of the buffered amplifier. This amplifier was designed to expose an input impedance of $Z_{i n}=65 \Omega$ that was determined to be the optimal one for maximal bandwidth of oscillation.

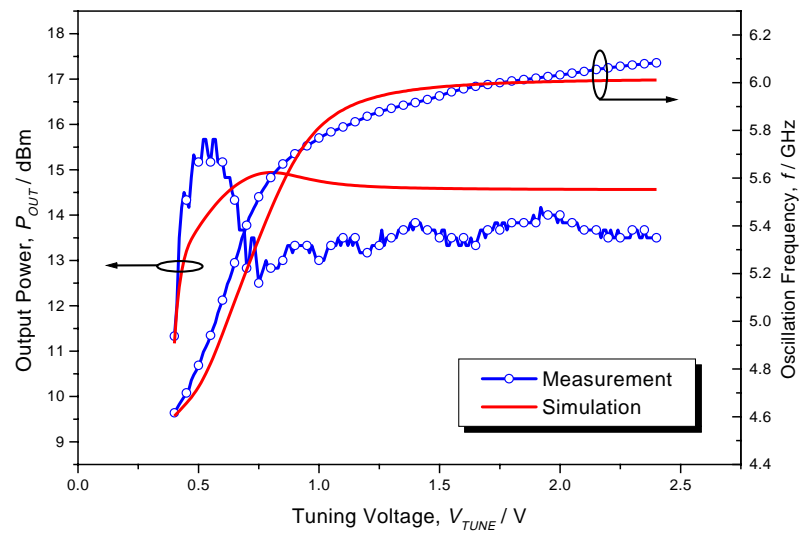

Fig. 4. Measured and simulated values of the VCO's oscillation frequency and output power versus negative tuning voltage

Figure 4 shows the simulated and measured variation of the oscillation frequency and output power versus applied tuning voltage for the final designs. The measured and simulated results agree well. The value of the frequency variation range of $1.48 \mathrm{GHz}$ with corresponding 
relative bandwidth of almost $28 \%$ is very high for a fully monolithically integrated VCO with simple diode tuning. In comparison to the unbuffered VCO, the bandwidth of oscillation could be again enlarged by over $4 \%$.

TABLE III

CALCULATED AND MEASURED CHARACTERISTICS OF TYPICAL BUFFERED OSCILLATOR

\begin{tabular}{|l|c|c|}
\hline & Sim. & Meas. \\
\hline \hline Frequency Range, $f / \mathrm{GHz}$ & $4.60-6.01$ & $4.61-6.09$ \\
\hline Rel. Bandwidth, $\Delta f / f_{m} / \%$ & 26.6 & 27.7 \\
\hline Output Power, $P_{\text {out }} / \mathrm{dBm}$ & $11.2-14.9$ & $11.2-15.5$ \\
\hline
\end{tabular}

Further simulations have shown that this value can still be increased, if the whole tuning range of the diodes (down to $V_{T U N E}=0 \mathrm{~V}$ ) is used. As listed in Table III and shown in Fig. 4, the output power of the buffered oscillator circuit demonstrates low variation versus tuning voltage and exhibits a high level of 11.2 up to $15.5 \mathrm{dBm}$.

Finally, Fig. 5 shows a microphotograph of the realised buffered oscillator. The VCO is very compact and requires area with dimensions of $2.5 \times 1.0 \mathrm{~mm}^{2}$.

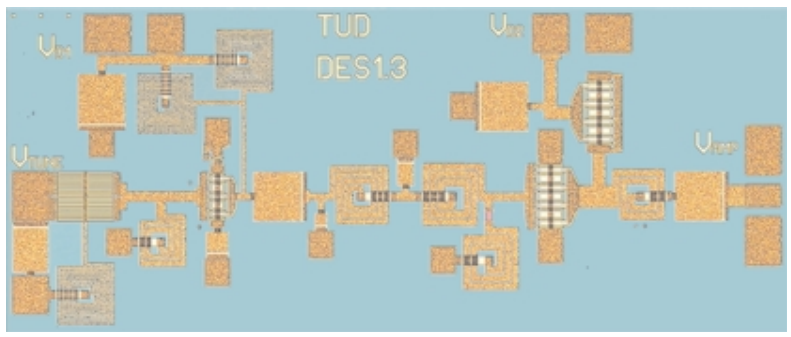

Fig. 5. Microphotograph of the buffered oscillator manufactured. Dimensions: $1.0 \times 2.5 \mathrm{~mm}^{2}$.

\section{CONCLUSIONS}

Special design solutions are introduced here that help to increase the bandwidth of oscillation of fully integrated MMIC-VCO realisations. These are in particular:

- a resonator configuration that is more suitable for the wide-band application. The effective capacitance modulation ratio is increased under large-signal conditions by means of series-parallel connection of integrated planar diodes.
- advanced active biasing technique that realise the separation of DC- and RF-paths in an increased frequency range in comparison to the traditional passive realisation. Furthermore, this solution allows to increase the DC to RF conversion efficiency of the circuits and to decrease the chip-area demand.

- it can be shown that by terminating the VCO circuitry with an appropriate load impedance (by means of buffering amplifiers with suitable input impedance) the range of oscillations can be increased.

At this point, it has to be emphasised that only by careful combinations of all the mentioned solutions the very good performance of the circuits described could be achieved.

Using the concepts developed, new tunability ranges of high output-power levels can be obtained by monolithic integration of voltage controlled oscillators. This represents, therefore, an important contribution to the many applications of MMICs from sensor to mobile communications.

\section{ACKNOWLEDGEMENTS}

This work has been funded by the ADAM OPEL AG, Rüsselsheim. Further, the authors wish to express their gratitude to the staff member of UMS for manufacturing the MMICs.

\section{REFERENCES}

[1] K. J. Anderson and D. L. Allen: "A Miniature Integrated Monolithic VCO Module", in IEEE MTT-S Dig., pp. 1503-1506, 1998.

[2] H. Q. Tserng, and H. M. Macksey, "Performance of a Monolithic GaAs FET Oscillators at J-Band," IEEE Trans. Electron Devices, Vol. ED-28, pp. 163-165, Feb. 1981.

[3] J. Portilla, M. L. de la Fuente, J. P. Pascual, and E. Artal, "LowNoise Monolithic Ku-Band VCO Using Pseudomorphic HEMT Technology," IEEE Microwave Guided Wave Lett., vol. 7, no. 11, pp. 380-382, Nov. 1997.

[4] M. Ahdjoudi, A. Boudiaf, and P. Pouvil, "A Pseudomorphic HEMT Monolithic K-Band VCO," in Proc. of 26th EuMC, Prague, pp. 101-103, Sept. 1996.

[5] Y. Cho, S. Hong, and Y. Kwon, "A Novel Active Inductor and Its Application to Inductance-Controlled Oscillator," IEEE Trans. Microwave Theory Techn., vol. MTT-45, no. 8, pp. 1208-1213, Aug. 1997.

[6] H. Hayashi, and M. Muragushi, "A Novel Broad- Band VCO Using an Active Inductor," IEICE Trans. Fundamentals, vol. E81-A, no. 2, pp. 224-229, Feb. 1998.

[7] T. Takenaki, A. Miyazaki, and H. Matsuura, "Wideband MMIC Voltage Controlled Oscillator Using Active Impedance Load Matching," in Proc. of IEEE MTT-S Dig., pp. 1503-1506, 1996. 